\title{
Future Perspective for Radiation Dose Reduction techniques in Computed Tomography - A Study
}

\section{Vivekanadam Balasubramaniam}

Faculty of Computer Science and Multimedia, Lincoln University College,

Malaysia.

Email: vivekanandam@lincoln.edu.my

\begin{abstract}
The Computed Tomography (CT) image quality is determining by appropriate radiation dose in CT examination. Increases of the radiation dose become dangerous for our health such as induces of cancer, skin injuries, heritable mutations, reddening, burn the skins, etc. Therefore, the dose management study in the CT scanning procedure is one of the most important factors. This research article focuses on the use of the dose effectively in pediatric CT and cardiac CT scan procedures. Besides, the paper comprises dose hunt-down, auditing the scanner utilization, patient safety for the hospital association. This research article discusses radiation dose reduction techniques for effective dose in the view of future perspective in CT scan. This research article suggesting an appropriate technique to reduce the dose effectively in CT images during scanning. The effective dose test was conducted after reviews and ideas from future perspective designs.
\end{abstract}

Keywords: Computed Tomography, Radiation risk, Radiation dose

\section{INTRODUCTION}

Computed Tomography is playing a vital role in medical fields for understanding and diagnosing various diseases. The use of CT is recognized in the ubiquitous, emerging application such as any type of analysis for organ function in our body [1]. During the last twenty years, the utilization of CT is increased in the medical field and provided suitable answers for many critical cases in the hospitals. The two CT scan procedures named pediatric CT and cardiac CT are using in more populated developed countries hospitals very commonly [2]. Recently, the radiology department focuses on dose management for improving patient safety. The ray beam lies on the patient's body includes motorized table, detectors so on which is shown in figure 1 . The measuring unit for radiation dose is Sievert (Sv) [3], [4]. First of all, the reduction of radiation dose for pediatric patient's to ignore or neglect unnecessary CT exams. Look for an alternative method is the best solution to reduce dose for pediatric patients [5], [6].

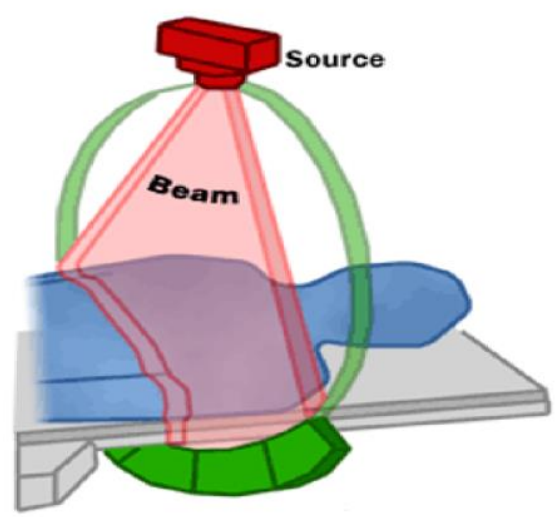

Figure 1 X-Ray source

The dose descriptors cannot be neglect for many reasons in the context due to quality outcomes with meaningful scanning. The dose descriptors are volume of CT dose index, dose length creation, effective dose, dose size estimation specifically. For the quality assessments, there is a need of checking manage doses and 
Journal of Innovative Image Processing (JIIP) (2020)

Vol.02/ No. 04

Pages: $210-216$

https://www.irojournals.com/iroiip/

DOI: https://doi.org/10.36548/jiip.2020.4.006

analyzing the quality of the dose [7]. This research article discusses methods of reducing dose in radiation. Figure 2 shows a picture of the CT scan examination procedure in the human body.

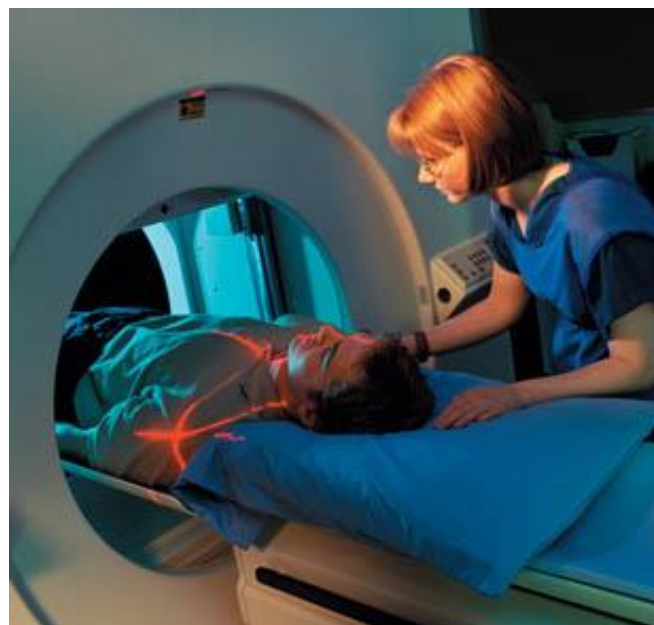

Figure 2 Picture of CT scan examination procedure

An effective dose management provides better auditing for patient safety and appropriate usage of the scanner. This availability can help in scrutinizing for dose efficiency of radiation doses in scanners [8]. The author strongly believes that there is a difference between technical data and real-time practice with the scanner. Always this practice will lead to dose tracking for the usage of dose in the scanner. The CT protocol is widely used by reduced doses in its examinations for diagnosis of disease [9]. The dose management system has attempted by collecting data manually from many communication systems form of picture archiving. To avoid typographic error in the radiology sector, the department concentrates to allow the dose management consistency and it is to be an effective process [10]. There is questionable patient safety when the hospital is used high-dose radiation in CT scans for higher quality images. Recently this dose management has grown in the medical field after the detrimental effects of an overdose in the radiation field. The various protocols with many optimization technical factors can able to activate this dose management system. This reasonable action can have visualized on the big data management that reflects inadequate conceptualize. We were stuck in some basic question in the radiation field before conducting the dose management system as follows;

\section{Research Motivation}

Now a day, the usage of CT scan in pediatric and cardiac in many clinical scenarios for accuracy reason. Also, special consideration should be made by medical rotary when using for child and old age people who are pathologically feeble. Therefore, the handling of CT scan with an effective dose during the examination procedure. The changes or decreases in radiation dose provide enough motivation for the operator's practice. The study for use of effective dose in the radiation field is a very essential stroke in the radiological sector. This research idea also is established based on a past review of radiology's literature paper.

\section{ORGANISATION OF RESEARCH ARTICLE}

The structure of the research article is organized as follows; Section 3 gives related work of recent research papers on radiation reduction techniques. Section 4 discusses radiation reduction methods and their facing problems and proposed a solution to the process for future perspectives. Section 5 delivers a description of effective dose for various examination, finally, the conclusion and the future task is in section 6 respectively.

\section{RELATED WORK}

Brenner DJ et al surveys 1.5 to $2 \%$ of cancers will occur due to radiation dose in CT [11]. This estimate is very highly debatable based on the National Academies of Science (NAS) report for ionizing radiation effects [12]. J Valentin discusses other radiation effects and risks to the organs of the human body during CT examinations [13]. Jacobi $\mathrm{W}$ introduces the concept of effective dose for every human body may change due to their condition of the body which is expressed by $\mathrm{mSv}$ [14] [15]. Also, McCollough $\mathrm{CH}$ et al explained about "whole body equivalent dose" based on their risk of health detriment and body irradiation [16]. 
Journal of Innovative Image Processing (JIIP) (2020)

Vol.02/ No. 04

Pages: $210-216$

https://www.irojournals.com/iroiip/

DOI: https://doi.org/10.36548/jiip.2020.4.006

The report having a comparison of radiation risk during different types of CT examination based on the dosage in radiation [17]. The effective dose can be determined by many factors such as relative hazard detriment of organs in the body, the organ's sensitivity to radiation. This will be calculated by a weighted sum of the corresponding doses for the organs in the human body [18]. DeMarco JJ et al, Perisinakis K et al, and Deak P et al, Myronakis $\mathrm{M}$ et al are developed a tool package for determining effective dose for every human body as named "patient-specific Monte Carlo" [19] [20] [21] [22]. The development of induced cancer is evaluating with a lowlevel dose of less than $100 \mathrm{mSV}$ discussed here [23]. Fletcher JG et al involves the detection of high contrast situations from the tagged stool in CT colonography [24]. Callstrom MR et al discuss high-level noise and even low-level dose are providing confidence in diagnostic property [25]. Hsieh discusses significant detection and geometrical efficiency in $\mathrm{x}$-ray detector proves the important contributing factor for the improved performance of CT scanner [26]. The article describes the measurement of radiation patterns and range of dose profile for detector module for bidirectional movement in the row [27]. Siewerdsen JH et al assessing and investigates dose usage and image quality for the optimization procedure of the design for the system [28].

It is known that there are three main classifications of usage of doses named as absorbed, equivalent, and effective dose. Based on the patient's conditions, the radiologist sets the dosage amount for any x-ray or CT scan.

\section{Research questions}

1. When does radiation dose can be reduced for flawless diagnostic image quality?

2. Why can we use the effective dose in radiation examination procedures at all times?

3. What are benefits can be obtained after usage of effective dose in the examination procedure?

\section{METHODOLOGY}

The following techniques are identified as radiation reduction methods for general dosage;

1. Fixed tube current (technique charts)

2. Tube current $(\mathrm{mA})$ modulation

3. Automatic exposure control (AEC)

4. Adjusting $\mathrm{kV}$ based on patient size - Iterative reconstruction

\subsection{Pediatric CT scan}

The scanning factor is designed for pediatric CT scans that should be very low. The whole protocol mechanism includes tube current modulation, radiation filter, scanning beam view that can be determined appropriate kilo Volt or milliAmpere for any system to the patients. It is called a size-based technique. Based on the low attenuation in the body, the system noise level will not increase with the voltage of the system. Therefore, the examination scan protocol can improve the scanning image quality without increase the noise level. The dose reduction will be happening significantly during this scenario. This method has been investigated and used in many pediatric patients [19] [29] [30] [31]. The following problems have encountered during the testing procedure.

\section{Existing Problem:}

This lower $\mathrm{kV}$ method practices are considering serval factors due to low beam x-ray from the tube gives increases of current to avoid excessive noise level. Also, some of the patient's body condition will not support this type of radiation dose examination.

\section{Proposed Solution:}

With the help of size based measurement method, we can solve the problem. Moreover, reduce motion artifacts in the scanning machine for pediatric patients is another procedure to solve the issue. Also, fast-moving time and high helical distance are appropriate during scanning to avoid the above problem. This parameter will limit the tube current usage in the scanner. Also, it will help to meet good CT examination quality. So this pediatric exam can be handled and evaluated by radiologists carefully.

\section{Measurement of risk estimation}

The pediatric CT procedure is estimated in terms of the range of 1 to $10 \mathrm{mSv}$ for the effective doses. The expert survivors are identified and demonstrated to determine the effective doses for CT procedure to reduce radiation dose. 
Journal of Innovative Image Processing (JIIP) (2020)

Vol.02/ No. 04

Pages: $210-216$

https://www.irojournals.com/iroiip/

DOI: https://doi.org/10.36548/jiip.2020.4.006

\subsection{Cardiac CT scan}

The handling of the dosage and dose reduction or increases in cardiac CT scan is a more complex issue compared to other applications. Due to different relationships in cardiac CT scans, the handling is a bit difficult in dose reduction. This dose reduction is depending on the patient's conditions and equipment specification too.

\section{Existing Problem:}

The high current values in the cardiac CT scanner system are directly proportional to pitch values (bit low). The handling of dose reduction is a bit difficult in the cardiac CT scanner machine due to the immobile condition of the beam and rays.

\section{Proposed Solution:}

The effective dose can handle the organ dose values by Monte-Carlo (MC) calculations during the CT examination procedure. Therefore, the tube current modulation method is the only way to reduce dose in cardiac CT scanner which is used to take Electro Cardio Gram (ECG). The one with the least motion is the best phase method to reduce the dose in radiation with the maximum current to get acquire the quality of cardiac CT examination.

\section{Measurement of risk estimation}

The risk estimates in cardiac CT is very dynamic because of continuous assessment of radiology procedure for CT scanner. The radiation-related risk is very low due to low dose tends directly proportional risk level. Many research paper concludes that low dose of radiation will not increase the risk of developing cancer,

\subsection{Future perspective}

Based on the above problem and solutions, the author is suggesting some following perspective of reduction of dose in future in radiology sector.

\section{Reconstruction scanning techniques}

The filtered-based reconstruction technique is one the best method to improve the image quality relative to existing methods [32]. Because of this scanning range is wide and categorized under the hybrid model. Also, this will use a very effective dose for radiation purposes. Also, this type of study is demonstrated for the dynamic noisy sector in CT imaging to get high contrast spatial domain [33]. This technique is maintaining less noise with high current concerning effective dose of filtered-based reconstruction techniques [32]. The method is permitting effective doses for different organs are irradiated.

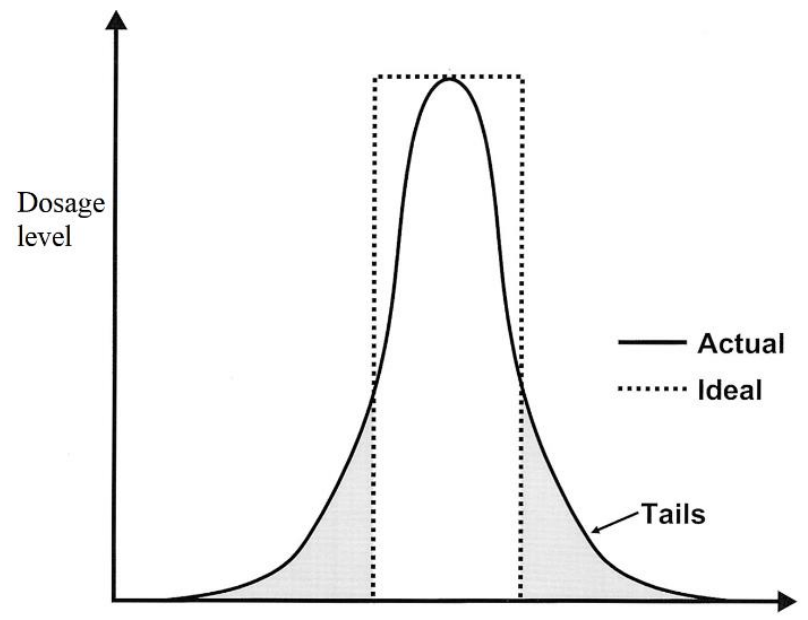

Figure 3 Radiation dosage in cardiac CT

This research article provides the highlight of some of the common cardiac CT imaging to encounter for radiologists. Figure 3 shows the appropriate radiation dosage in cardiac CT scan.

\section{RESULTS AND DISCUSSION}

During the time of giving up the image quality of the scan report, the radiation dose can be reduced as much as possible. Therefore, the full-fledged flawless diagnostic can be possible during good image quality due 
Journal of Innovative Image Processing (JIIP) (2020)

Vol.02/ No. 04

Pages: $210-216$

https://www.irojournals.com/iroiip/

DOI: https://doi.org/10.36548/jiip.2020.4.006

to high radiation dose. The effective dose can respond to flawless diagnostic examination. The distinctive of effective dose usage is the most handling method for many patients to reduce the radiation dose. There is no need of determining the dosage amount for every patient's condition in an effective dose method. Our research paper focuses on pediatric CT imaging for children where potential effects on radiation exist in the scanner.

Table 1 Results obtained from examination for effective dose

\begin{tabular}{|c|c|c|}
\hline S.No & Diagnostic Examination & Effective dose (mSv) \\
\hline 1 & Chest X-Ray & 0.0199 \\
\hline 2 & Spine & 1.52 \\
\hline 3 & Barium enema & 5 \\
\hline 4 & CT head position & 2 \\
\hline 5 & CT Chest Position & 7 \\
\hline 6 & CT abdomen position & 10 \\
\hline 7 & CT angiogram & 15 \\
\hline
\end{tabular}

The improper handling of pediatric CT scans with high dose or not measured dose leads to childhood cancer or organ malformation. But the effective dose in the examination procedure is used to reduce radiation risk for the human body due to the appropriate dosage level. Because children's body organs are very sensitive to radiation impairment. Figure 4 shows the nominal beam with for effective dose of CT scan.

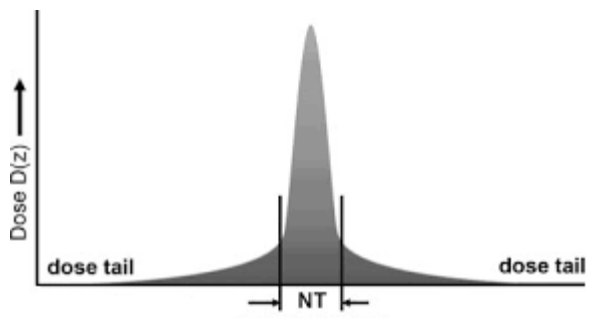

Figure 4 Setting the effective dose in CT radiation scanner

The table 2 contains results obtained from examination with the effective dose for appropriate diagnostic examination which is shown in table1.

Table 2 Results obtained from examination with the effective dose

\begin{tabular}{|c|l|l|}
\hline $\begin{array}{c}\text { Parameters affecting dose } \\
\text { reduction }\end{array}$ & \multicolumn{1}{|c|}{ Effect on image quality } & \multicolumn{1}{c|}{ Remedies } \\
\hline Tube current (mAs) & $\begin{array}{l}\text { The Image noise is indirectly } \\
\text { proportional to radiation dose. }\end{array}$ & $\begin{array}{l}\text { The noise threshold level } \\
\text { examination for CT }\end{array}$ \\
\hline Tube voltage $(\mathrm{kVp})$ & $\begin{array}{l}\text { Obtaining low contrast resolution } \\
\text { in image quality based on } \\
\text { radiation dose. }\end{array}$ & Determining the effective dose \\
\hline Helical pitch & $\begin{array}{l}\text { The increment of noise is due to in } \\
\text { The pitch distance is directly } \\
\text { proportional to noise variation. }\end{array}$ & $\begin{array}{l}\text { The effective adjustment in } \\
\text { collimation pitch between } 40- \\
82 \mathrm{~mm} .\end{array}$ \\
\hline Scan range & $\begin{array}{l}\text { The scan length is directly } \\
\text { proportional to dosage }\end{array}$ & $\begin{array}{l}\text { Set the scan range appropriate } \\
\text { with dosage. }\end{array}$ \\
\hline Automatic exposure control & $\begin{array}{l}\text { The adjustment of dose based on } \\
\text { the patient's conditions. }\end{array}$ & $\begin{array}{l}\text { The modulation current is } \\
\text { compensating of the tube to } \\
\text { detector. }\end{array}$ \\
\hline Temporal resolution & $\begin{array}{l}\text { Comparison of the patient's heart } \\
\text { beat rate }\end{array}$ & The decrement of resolution \\
\hline $\begin{array}{c}\text { Our proposed } \\
\text { Reconstruction Scanning } \\
\text { Techniques }\end{array}$ & $\begin{array}{l}\text { The consistency pattern is directly } \\
\text { depending on the tube current. }\end{array}$ & $\begin{array}{l}\text { The filtered based projection } \\
\text { increases for the model }\end{array}$ \\
\hline
\end{tabular}

ISSN: 2582-4252 (online) 
Journal of Innovative Image Processing (JIIP) (2020)

Vol.02/ No. 04

Pages: $210-216$

https://www.irojournals.com/iroiip/

DOI: https://doi.org/10.36548/jiip.2020.4.006

Radiation protection is a traditional approach that is used in many CT scan examinations. The risk from cancer due to excess radiation in the system is proportional to the amount of radiation dose absorbed by the body.

\section{CONCLUSION}

This research article covered the topics of reduction of radiation dose techniques in CT scans. The author strongly believes that this research article helps many radiologists to set the radiation dose during pediatric CT and cardiac CT examination procedures. Because the number of CT examinations increases recently, handling the radiation dose very random. According to the specific measurement of radiation dose for the CT examination and patient's condition, the automatic control facilities will be developed further based on the future perspective of the iteration technique. The optimization of the radiation dose with dynamic tube current enables the hybrid adjustment to give a reduction of radiation dose efficiently. The organ motion analysis is done under by reduction of radiation dose methods in the radiologic image. The radiologist is acquiring the imaging with the various techniques and to ensure the radiation exposure is low as much as possible without comprising of image quality. The author will be focused this problem statement as a future task of research.

\section{REFERENCES}

[1] Brenner DJ, Hricak H. Radiation exposure from medical imaging: time to regulate? JAMA 2010;304(2):208209.

[2] Gottumukkala RV, Kalra MK, Tabari A, Otrakji A, Gee MS. Advanced CT Techniques for Decreasing Radiation Dose, Reducing Sedation Requirements, and Optimizing Image Quality in Children. Radiographics. 2019 May-Jun;39(3):709-726. doi: 10.1148/rg.2019180082. Epub 2019 Mar 29. PMID: 30924753.

[3] Sabarudin A, Sun Z. Coronary CT angiography: Dose reduction strategies. World J Cardiol. 2013 Dec 26;5(12):465-72. doi: 10.4330/wjc.v5.i12.465. PMID: 24392191; PMCID: PMC3879694.

[4] Farooque MC, Ashraf Ahmed B, Abubacker Sulaiman F, Anand Rajamani, Gopal Krishnan, Divya Y. Method of reducing the radiation dose to the paediatric age groups in computed tomography. International Journal of Contemporary Medicine Surgery and Radiology. 2019;4(3):C263-C266.

[5] Rawashdeh MA, Saade C. Radiation dose reduction considerations and imaging patterns of ground glass opacities in coronavirus: risk of over exposure in computed tomography. Radiol Med. 2020 Sep 8:1-8. doi: 10.1007/s11547-020-01271-2. Epub ahead of print. PMID: 32897493; PMCID: PMC7477737.

[6] Kubo T, Lin PJ, Stiller W, Takahashi M, Kauczor HU, Ohno Y, Hatabu H. Radiation dose reduction in chest CT: a review. AJR Am J Roentgenol. 2008 Feb;190(2):335-43. doi: 10.2214/AJR.07.2556. PMID: 18212218.

[7] Yu L, Liu X, Leng S, Kofler JM, Ramirez-Giraldo JC, Qu M, Christner J, Fletcher JG, McCollough CH. Radiation dose reduction in computed tomography: techniques and future perspective. Imaging Med. 2009 Oct;1(1):65-84. doi: 10.2217/iim.09.5. PMID: 22308169; PMCID: PMC3271708.

[8] McCollough CH, Primak AN, Braun N, Kofler J, Yu L, Christner J. Strategies for reducing radiation dose in CT. Radiol Clin North Am. 2009 Jan;47(1):27-40. doi: 10.1016/j.rcl.2008.10.006. PMID: 19195532; PMCID: PMC2743386.

[9] Lukasiewicz A, Bhargavan-Chatfield M, Coombs L, et al. Radiation dose index of renal colic protocol CT studies in the United States: a report from the American College of Radiology National Radiology Data Registry. Radiology 2014;271(2):445-451.

[10] AlSuwaidi JS, AlBalooshi LG, AlAwadhi HM, et al. Continuous monitoring of CT dose indexes at Dubai Hospital. AJR Am J Roentgenol 2013;201(4):858-864.

[11] Brenner DJ, Hall EJ. Computed tomography - an increasing source of radiation exposure. N. Engl. J. Med. 2007; 357:2277-2284. [PubMed: 18046031] - Estimates the lifetime risk of cancer mortality attributable to radiation exposure in computed tomography $(\mathrm{CT})$. It received substantial media and public attention, despite the questionable methodology and conclusion.

[12] Biologic Effects of Ionizing Radiation (BEIR) Report VII: Health risks from exposure to low levels of ionizing radiation. National Academies. 2006.

[13] J Valentin, International Commission on Radiological Protection: Managing patient dose from multi detector computed tomography (MDCT), (ICRP Publication 102). Ann. ICRP. 2007; 37(1):1-79.

[14] Jacobi W. The concept of the effective dose - a proposal for the combination of organ doses. Radiat. Environ. Biophys. 1975; 12:101-109. [PubMed: 1178826]

[15] International Commission on Radiological Protection. Recommendations of the International Commission on Radiological Protection (ICRP Publication 26). Oxford, UK: The International Commission on Radiological Protection; 1977.

ISSN: 2582-4252 (online)

Submitted: 10.12 .2020

Revised: 18.01.2021

Accepted: 08.02.2021

Published: 22.02.2021 
Journal of Innovative Image Processing (JIIP) (2020)

Vol.02/ No. 04

Pages: $210-216$

https://www.irojournals.com/iroiip/

DOI: https://doi.org/10.36548/jiip.2020.4.006

[16] McCollough CH, Schueler BA. Calculation of effective dose. Med. Phys. 2000; 27:828-837. [PubMed: 10841384]

[17] International Commission on Radiological Protection: 1990 Recommendations of the International Commission on Radiological Protection (Report 60). Ann. ICRP. 1991; 21:1-3.

[18] International Commission on Radiological Protection: 2007 recommendations of the International Commission on Radiological Protection (ICRP Publication 103). Ann. ICRP. 2007; 37:1-332.

[19] DeMarco JJ, Cagnon CH, Cody DD, et al. Estimating radiation doses from multidetector CT using Monte Carlo simulations: effects of different size voxelized patient models on magnitudes of organ and effective dose. Phys. Med. Biol. 2007; 52:2583-2597. [PubMed: 17440254]

[20] Perisinakis K, Tzedakis A, Damilakis J. On the use of Monte Carlo-derived dosimetric data in the estimation of patient dose from CT examinations. Med. Phys. 2008; 35:2018-2028. [PubMed: 18561678]

[21] Deak P, van M Straten, Shrimpton PC, Zankl M, Kalender WA. Validation of a Monte Carlo tool for patientspecific dose simulations in multi-slice computed tomography. Eur. Radiol. 2008; 18:759-772. [PubMed: 18066555]

[22] Myronakis M, Perisinakis K, Tzedakis A, Gourtsoyianni S, Damilakis J. Evaluation of a patientspecific Monte Carlo software for CT dosimetry. Radiat. Prot. Dosimetry. 2009; 133:248-255. [PubMed: 19329513]

[23] National Research Council (US) Committee on Health Effects of Exposure to Low Levels of Ionizing Radiations (BEIR VII). Health Effects of Exposure to Low Levels of Ionizing Radiations: Time for Reassessment? Washington (DC): National Academies Press (US); 1998. Available from: https://www.ncbi.nlm.nih.gov/books/NBK230461/ doi: 10.17226/6230

[24] Fletcher JG, Johnson CD, Welch TJ, et al. Optimization of CT colonography technique: prospective trial in 180 patients. Radiology. 2000; 216:704-711. [PubMed: 10966698]

[25] Callstrom MR, Johnson CD, Fletcher JG, et al. CT colonography without cathartic preparation: feasibility study. Radiology. 2001; 219:693-698. [PubMed: 11376256]

[26] Hsieh, J. Computed Tomography: Principles, Design, Artifacts, and Recent Advances. Bellingham, WA, USA: SPIE Press; 2006.

[27] International Electrotechnical Commission. Medical electrical equipment. Part 2-44: Particular requirements for the safety of x-ray equipment for computed tomography. IEC publication No. 60601-60602-60644. Ed. 2.1. Geneva, Switzerland: International Electrotechnical Commission (IEC); 2002.

[28] Siewerdsen JH, Jaffray DA. Cone-beam computed tomography with a flat-panel imager: magnitude and effects of X-ray scatter. Med. Phys. 2001; 28:220-231. [PubMed: 11243347]

[29] van der Molen AJ, Geleijns J. Overranging in multisection CT: quantification and relative contribution to dose - comparison of four 16-section CT scanners. Radiology. 2007; 242:208-216. [PubMed: 17090713]

[30] Nakayama Y, Awai K, Funama Y, et al. Abdominal CT with low tube voltage: preliminary observations about radiation dose, contrast enhancement, image quality, and noise. Radiology. 2005; 237:945-951. [PubMed: 16237140]

[31] Schindera ST, Nelson RC, Mukundan S Jr, et al. Hypervascular liver tumors: low tube voltage, high tube current multi-detector row CT for enhanced detection - phantom study. Radiology. 2008; 246:125-132. [PubMed: 18096533]

[32] Higuchi K et al (2013) Detection of ground-glass opacities by use of hybrid iterative reconstruction (iDose) and low-dose 256-section computed tomography: a phantom study. Radiol Phys Technol 6(2):299-304.

[33] Pontana F et al (2011) Chest computed tomography using iterative reconstruction vs fltered back projection (Part 1): evaluation of image noise reduction in 32 patients. Eur Radiol 21(3):627-635.

ISSN: 2582-4252 (online)

Submitted: 10.12.2020

Revised: 18.01.2021

Accepted: 08.02.2021 